\title{
Loss of the Normal Gradient in Arterial Compliance and Outcomes of Chronic Kidney Disease Patients
}

\author{
Qiong Bai Chun-Yan Su Ai-Hua Zhang Tao Wang Wen Tang \\ Department of Nephrology, Peking University Third Hospital, Beijing, China
}

\section{Keywords}

Arteries stiffness $\cdot$ Pulse wave velocity ratio $\cdot$ Chronic kidney disease $\cdot$ Outcome

\begin{abstract}
Background: In dialysis patients, loss of the normal gradient in arterial compliance, assessed by the pulse wave velocity (PWV) ratio, predicts all-cause mortality better than does carotidfemoral PWV (CF-PWV) alone. However, the prognostic significance of the PWV ratio for outcome in chronic kidney disease (CKD) patients remains unclear. Methods: In this longitudinal cohort study, CKD patients who visited our CKD management clinic between April 27, 2006, and March 27, 2008, were included and followed up. To assess the gradient in arterial compliance, the PWV ratio was calculated using CF-PWV divided by carotid-radial PWV. Results: A total of 209 patients in CKD stages 1-4 with a median follow-up of 3.74 years were included. Patients with higher PWV ratio were relatively older $(p<0.001)$ and had worse renal function $(p<0.001)$, more hypertension $(p<0.001)$, diabetes mellitus $(p<0.001)$, and cardiovascular or cerebrovascular disease $(p<0.001)$. The median time to patient outcome (death, renal replacement therapy, or double increase in serum creatinine from baseline) in the group with a PWV ratio above the median (89.8 months, $95 \% \mathrm{Cl} 84.2-95.5)$ was shorter than that in the group with a PWV ratio below the median (105.3 months, 95\% Cl 101.3-109.3, $p=0.001)$. Univariate Cox regression analysis showed that both PWV ratio and CF-PWV were significantly associated with patient outcome. In multivariate Cox regression analysis, both PWV ratio and CF-PWV were associated with patient outcome. However, the HR for CF-PWV $(2.177,95 \% \mathrm{Cl}$ $1.064-4.453, p=0.033)$ was slightly higher than that for PWV ratio $(2.091,95 \% \mathrm{Cl} 1.049-4.167$, $p=0.036)$. There was a significant interaction effect between PWV ratio and CKD stage. It was
\end{abstract}


shown that patients with advanced CKD stages and higher PWV ratios had a significantly higher risk of adverse CKD outcome $(p=0.006)$. Conclusions: The PWV ratio, as a measure of loss of the normal gradient in arterial compliance, was associated with CKD patient outcome. Patients with advanced CKD and a higher PWV ratio had a significantly higher risk of adverse CKD outcome.

(c) 2019 S. Karger AG, Basel

\section{Introduction}

Chronic kidney disease (CKD) is a global healthcare burden affecting millions of individuals worldwide, associated with adverse health outcomes and high healthcare costs $[1,2]$. CKD increases the risks for cardiovascular disease (CVD) and all-cause mortality [3-6]. Arterial stiffness, defined as a decreased ability of the conducting arteries to absorb pulse pressure, is an independent predictor of cardiovascular morbidity and mortality in the general population and among CKD patients [7-10]. Our previous studies also demonstrated that arterial stiffness was associated with many CVD risk factors in peritoneal dialysis (PD) patients [11-18]. In addition, arterial stiffening occurs in parallel with a decline in glomerular filtration rate (GFR) and is associated with CKD progression [19].

Pulse wave velocity (PWV) is an indirect measure of stiffness and is the accepted standard for the noninvasive assessment of aortic stiffness [10]. Increased aortic stiffness, most commonly measured by carotid-femoral PWV (CF-PWV), has been widely used to demonstrate the predictive value of aortic stiffness for cardiovascular events [9, 10]. Although CF-PWV is considered the "gold standard" for measuring aortic stiffness, some recent studies have indicated that the PWV ratio could be a better indicator than CF-PWV [20-22].

The PWV ratio (CF-PWV divided by carotid-radial PWV [CR-PWV]) is a relatively new concept in arterial research [20,22-26]. Under physiological conditions, the arterial vasculature is characterized by a progressive increase in stiffness from the aorta and large elastic arteries toward the peripheral muscular conduit arteries, often labeled as the "arterial stiffness gradient" $[22,24]$. Reversal of the normal arterial stiffness gradient, as assessed by the PWV ratio, enhances the transmission of pulsatile energy into the periphery and microcirculation and, theoretically, increases the risk for damage to microvascular beds in highly perfused organs [20, 22]. Fortier et al. [21] demonstrated that the PWV ratio was a better prognostic predictor of all-cause mortality than CF-PWV in a prevalent dialysis cohort (245 hemodialysis and 65 PD patients). Our latest research also demonstrated that the discriminative power of the PWV ratio for both all-cause and CVD mortality was better than that of CF-PWV in PD patients [27]. However, the findings from a recent study do not support the notion that the PWV ratio provides an additional prognostic value over conventional CF-PWV in the community [24]. Thus, the prognostic value of the PWV ratio for CKD patients needs to be confirmed by further study.

In the present study, we analyzed patients in CKD stages 1-4 with long follow-up periods and tried to investigate the predictive value of the PWV ratio with regard to all-cause mortality and the outcomes of renal deterioration and renal replacement therapy (RRT) in this cohort.

\section{Subjects and Methods}

In this longitudinal cohort study, a total of 616 CKD patients who visited our CKD management clinic between April 27, 2006, and March 27, 2008 were followed up. Patients with the following characteristics were excluded from data analysis: (1) paralysis or cognitive impairment following a cerebrovascular event; 
Bai et al.: PWV Ratio and CKD Outcomes

(2) absence of a femoral or radial pulse; (3) unwillingness to participate; (4) clinical instability like acute heart failure; or (5) malignant disease. A total of 209 patients in CKD stages 1-4 were included in this study.

The outcome in the present study was either all-cause mortality, any RRT (hemodialysis, PD, or renal transplantation), or a double increase in serum creatinine from baseline.

Measurement of PWV

All the PWV measurements were performed by one doctor, and the intra-observer coefficient of variation was about 5\%, as previously reported [17]. Aortic PWV was determined using an automatic device, the Complior (Colson, Garges-lès-Gonesses, France) [28], which allows online pulse wave recording and automatic calculation of PWV. Common carotid artery and femoral artery pressure waveforms were recorded noninvasively using a TY-306 Fukuda pressure-sensitive transducer (Fukuda, Tokyo, Japan) while the patient was in the supine position. Measurement was repeated over 10 different cardiac cycles, and the mean value was used for the final analysis. The distance traveled by the pulse wave was measured over the body surface as the distance between the two recording sites (D), while the pulse transit time ( $\mathrm{t}$ ) was automatically determined by the Complior device. PWV was automatically calculated as PWV = D/t. CF-PWV and CR-PWV were calculated from tonometry waveforms and body surface measurements, which were adjusted for parallel transmission in the brachiocephalic artery and aortic arch with the use of the suprasternal notch as a fiducial point. Loss of the normal gradient in arterial compliance (PWV ratio) was assessed using CF-PWV divided by CR-PWV.

Measurement of Blood Pressure

Blood pressure (BP) was measured when doing the PWV recordings. To ensure the accuracy of the measurements, a dedicated nephrology specialist nurse was in charge of all BP measurements, and the mercury sphygmomanometer used was calibrated regularly. All measurements were performed in a quiet room. Brachial BP was measured twice with the patients in the sitting position after they had rested more than $10 \mathrm{~min}$. Systolic and diastolic BP were averaged from the values of the two measurements.

\section{Measurement of Biochemical Parameters}

Serum albumin was determined with the bromocresol green method. Other biochemical indices, such as blood urea nitrogen and serum creatinine, were determined by standard methods. Patients' estimated GFR (eGFR) was estimated with the Chronic Kidney Disease Epidemiology Collaboration (CKD-EPI) creatinine equation [29].

\section{Statistical Analysis}

Results are expressed as frequencies and percentages for categorical variables; the mean \pm standard deviation for continuous, normally distributed variables; and the median (interquartile range) for continuous variables that were not normally distributed. Comparisons between patients in different groups were performed using $\chi^{2}$ tests, two-tailed unpaired $t$ tests, or Mann-Whitney tests, depending on data distribution. Time to event analysis was evaluated by Kaplan-Meier and multivariate Cox proportional-hazards survival analyses. The multivariate Cox proportional-hazards model included all the significant variables $(p<0.1)$ from the univariate analysis.

Statistical analysis was performed using IBM SPSS Statistics software (version 25.0). $p$ values $<0.05$ were considered statistically significant.

\section{Results}

\section{Baseline Characteristics of the Studied Population}

All the patients were followed up until December 31, 2015. A total of 209 patients in CKD stages 1-4 with a median follow-up of 3.74 years (interquartile range 2.50-5.65) were included in the present study. The patients were divided into two groups according to the median PWV ratio: a group below the median $(n=103)$ and a group above the median $(n=$ 106). The baseline comparison between the two groups is depicted in Table 1. Patients with a PWV ratio above the median had a significantly higher CF-PWV (12.16 \pm 2.12 vs. 9.12 \pm 1.17 , 
Bai et al.: PWV Ratio and CKD Outcomes

Table 1. Clinical parameters of all patients at baseline according to the median PWV ratio

\begin{tabular}{|c|c|c|c|}
\hline \multirow[t]{2}{*}{ Variables } & \multicolumn{3}{|l|}{ PWV ratio } \\
\hline & $\begin{array}{l}\text { below median } \\
(n=103)\end{array}$ & $\begin{array}{l}\text { above median } \\
(n=106)\end{array}$ & $p$ value \\
\hline \multicolumn{4}{|l|}{ PWV } \\
\hline CF-PWV & $9.12 \pm 1.17$ & $12.16 \pm 2.12$ & $<0.001$ \\
\hline CR-PWV & $9.66 \pm 1.63$ & $8.89 \pm 1.59$ & $<0.001$ \\
\hline PWV ratio & $0.95 \pm 0.11$ & $1.40 \pm 0.36$ & $<0.001$ \\
\hline $\mathrm{SBP}, \mathrm{mm} \mathrm{Hg}$ & $125 \pm 15$ & $138 \pm 20$ & $<0.001$ \\
\hline $\mathrm{DBP}, \mathrm{mm} \mathrm{Hg}$ & $79 \pm 11$ & $80 \pm 13$ & 0.169 \\
\hline Age, years & $55 \pm 16$ & $70 \pm 9$ & $<0.001$ \\
\hline Gender - male/female & $56 / 47$ & $50 / 56$ & 0.334 \\
\hline BMI, $\mathrm{kg} / \mathrm{m}^{2}$ & $24.2 \pm 3.55$ & $23.4 \pm 3.41$ & 0.877 \\
\hline Primary disease & & & $<0.001$ \\
\hline Glomerulonephritis or nephropathy & $46(44.2)$ & $32(30.5)$ & \\
\hline Ischemic disease & $19(18.3)$ & $48(45.7)$ & \\
\hline CIN & $17(16.4)$ & $15(14.3)$ & \\
\hline Cystic kidney disease & $3(2.9)$ & $3(2.8)$ & \\
\hline Unknown & $19(18.3)$ & $7(6.7)$ & \\
\hline Creatinine, $\mu \mathrm{mol} / \mathrm{L}$ & $140.7 \pm 54.5$ & $152.6 \pm 47.8$ & 0.093 \\
\hline Urea, $\mathrm{mmol} / \mathrm{L}$ & $8.27 \pm 3.31$ & $9.63 \pm 6.15$ & 0.053 \\
\hline Albumin, g/L & $42.6 \pm 5.58$ & $42.9 \pm 3.77$ & 0.708 \\
\hline Uric acid, $\mu \mathrm{mol} / \mathrm{L}$ & $392.6 \pm 120.1$ & $376.0 \pm 113.4$ & 0.325 \\
\hline Calcium, mmol/L & $2.24 \pm 0.14$ & $2.25 \pm 0.10$ & 0.607 \\
\hline Phosphorus, mmol/L & $1.22 \pm 0.20$ & $1.21 \pm 0.18$ & 0.700 \\
\hline eGFR, $\mathrm{mL} / \mathrm{min} / 1.73 \mathrm{~m}^{2}$ & $51.2 \pm 24.9$ & $38.8 \pm 16.0$ & $<0.001$ \\
\hline CKD stage & & & 0.001 \\
\hline 1 & $11(10.7)$ & 0 & \\
\hline 2 & $20(19.4)$ & $10(9.4)$ & \\
\hline $3 a$ & $21(20.4)$ & $19(17.9)$ & \\
\hline $3 b$ & $30(29.1)$ & $44(41.5)$ & \\
\hline 4 & $21(20.4)$ & 33 (31.1) & \\
\hline $1-3 a$ & $52(50.5)$ & $29(27.4)$ & \\
\hline $3 b-4$ & $51(49.5)$ & $77(72.6)$ & \\
\hline Total cholesterol, mmol/L & $5.25 \pm 1.76$ & $5.09 \pm 1.02$ & 0.46 \\
\hline Triglyceride, mmol/L & $2.21 \pm 1.45$ & $1.92 \pm 0.88$ & 0.148 \\
\hline $\mathrm{HDL}-\mathrm{c}, \mathrm{mmol} / \mathrm{L}$ & $1.53 \pm 0.55$ & $1.37 \pm 0.32$ & 0.029 \\
\hline LDL-c, mmol/L & $3.14 \pm 1.51$ & $3.13 \pm 0.91$ & 0.942 \\
\hline \multicolumn{4}{|l|}{ Medical history } \\
\hline Hypertension & $64(62.1)$ & $90(84.9)$ & $<0.001$ \\
\hline Diabetes mellitus & $11(10.7)$ & $35(33.0)$ & $<0.001$ \\
\hline Cardiovascular or cerebrovascular disease & $20(19.4)$ & $45(42.5)$ & $<0.001$ \\
\hline
\end{tabular}

Values are presented as the mean \pm SD or $n(\%)$. PWV, pulse wave velocity; CF-PWV, carotid-femoral PWV; CR-PWV, carotid-radial PWV; PWV ratio, CF-PWV divided by CR-PWV; SBP, systolic blood pressure; DBP, diastolic blood pressure; BMI, body mass index; CIN, chronic interstitial nephritis; eGFR, estimated glomerular filtration rate; CKD, chronic kidney disease; HDL-c, high-density lipoprotein cholesterol; LDL-c, low-density lipoprotein cholesterol.

$p<0.001)$ but a lower CR-PWV (8.89 \pm 1.59 vs. $9.66 \pm 1.63, p<0.001)$ than patients with a PWV ratio below the median. The eGFR was lower in the group with a PWV ratio above the median than in the group with a PWV ratio below the median (38.8 \pm 16.0 vs. $51.2 \pm 24.9 \mathrm{~mL} /$ $\left.\min / 1.73 \mathrm{~m}^{2}, p<0.001\right)$. PWV ratio was associated with CKD stage $(r=0.286, p<0.001)$. Besides, patients in the group with a PWV ratio above the median were relatively older $(p<$ $0.001)$ and had more hypertension $(p<0.001)$, diabetes mellitus $(p<0.001)$, and cardiovascular or cerebrovascular disease $(p<0.001)$ and a lower high-density lipoprotein cholesterol 
Bai et al.: PWV Ratio and CKD Outcomes

Fig. 1. Kaplan-Meier survival estimates for chronic kidney disease stage 1-4 patients in two different pulse wave velocity (PWV) ratio groups.

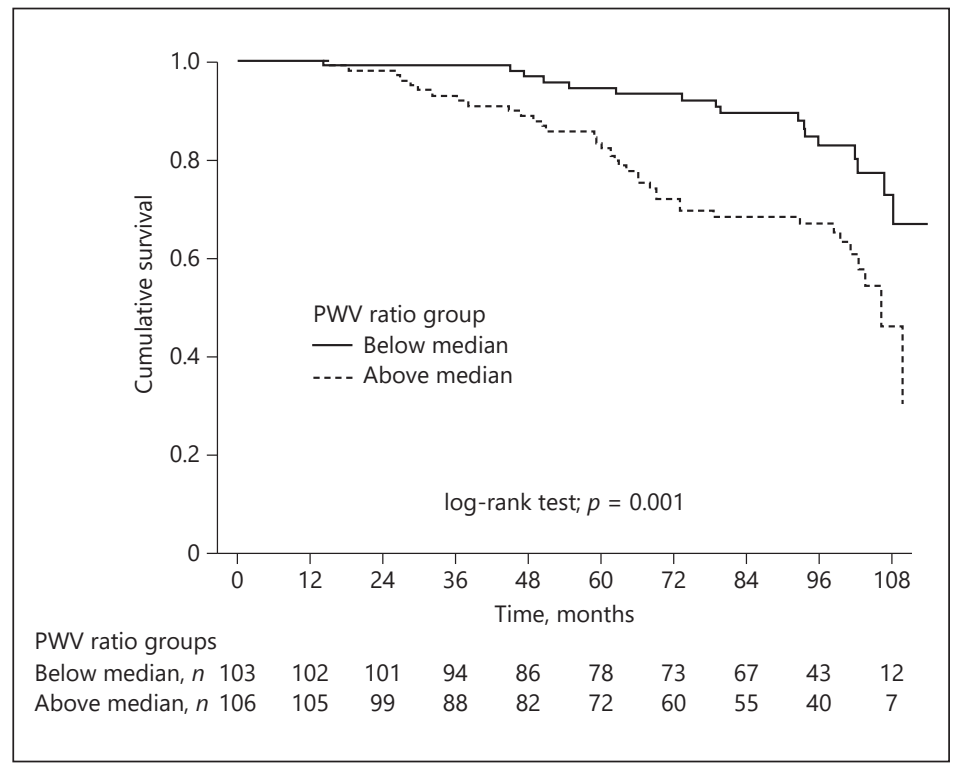

level ( $p=0.029)$. There was no significant difference in levels of serum creatinine, urea, albumin, uric acid, calcium, phosphorus, total cholesterol, triglyceride, low-density lipoprotein cholesterol, and BMI between the two groups.

\section{Factors Associated with CKD Patient Outcome}

Seventeen patients $(8.1 \%)$ died. A total of 30 patients $(14.3 \%)$ received RRT during follow-up (hemodialysis, 17 patients; PD, 12 patients; and renal transplantation,1 patient). A double increase in serum creatinine from baseline occurred in 29 patients $(13.9 \%)$.

The median time to patient outcome (death, RRT, or double increase in serum creatinine from baseline) was shorter in the group with a PWV ratio above the median (89.8 months, 95\% CI 84.2-95.5) than in the group with a PWV ratio below the median (105.3 months, 95\% CI 101.3-109.3, $p=0.001$ ) (Fig. 1).

The univariate Cox regression analysis showed that both PWV ratio (HR 2.603, 95\% CI 1.467-4.618, $p=0.001$ ) and CF-PWV (HR 1.751, 95\% CI 1.551-4.871, $p=0.001$ ) were significantly associated with patient outcome. Other factors associated with patient outcome were history of hypertension $(p=0.033)$, diabetes mellitus ( $p=0.007)$, cardiovascular or cerebrovascular disease ( $p=0.050)$, and CKD stage (CKD stages 3b-4 vs. CKD stages $1-3 a, p<0.001$ ).

To investigate the independent predictive value of PWV ratio for patient outcome, two multivariate Cox regression models were used (model 1 and model 2) (Table 2). In multivariate Cox regression model 1, we included PWV ratio, hypertension, diabetes mellitus, cardiovascular or cerebrovascular disease, CKD stage, and age. It was found that PWV ratio (HR 2.091, 95\% CI 1.049-4.167, $p=0.036$ ) was independently and significantly associated with patient outcome in model 1. In multivariate Cox regression model 2, we included CF-PWV, hypertension, diabetes mellitus, cardiovascular or cerebrovascular disease, CKD stage, and age. CF-PWV was a significant and independent predictor of patient outcome (HR 2.177, 95\% CI 1.064-4.452, $p=0.033$ ). However, compared with the HR for PWV ratio in model 1, the HR for CF-PWV was slightly higher. The interaction of PWV ratio and CKD stage was investigated. The predictor effect of PWV ratio was further investigated by stratifying the patients according to CKD stage and PWV ratio (model 3; Table 2). The results showed that patients with advanced CKD and a higher PWV ratio had a significantly higher risk of adverse CKD outcome. 
CardioRenal

Medicine
Cardiorenal Med 2019;9:297-307

\begin{tabular}{l|ll}
\hline DOI: 10.1159/000500479 & C 2019 S. Karger AG, Basel \\
\hline
\end{tabular}

www.karger.com/crm

Bai et al.: PWV Ratio and CKD Outcomes

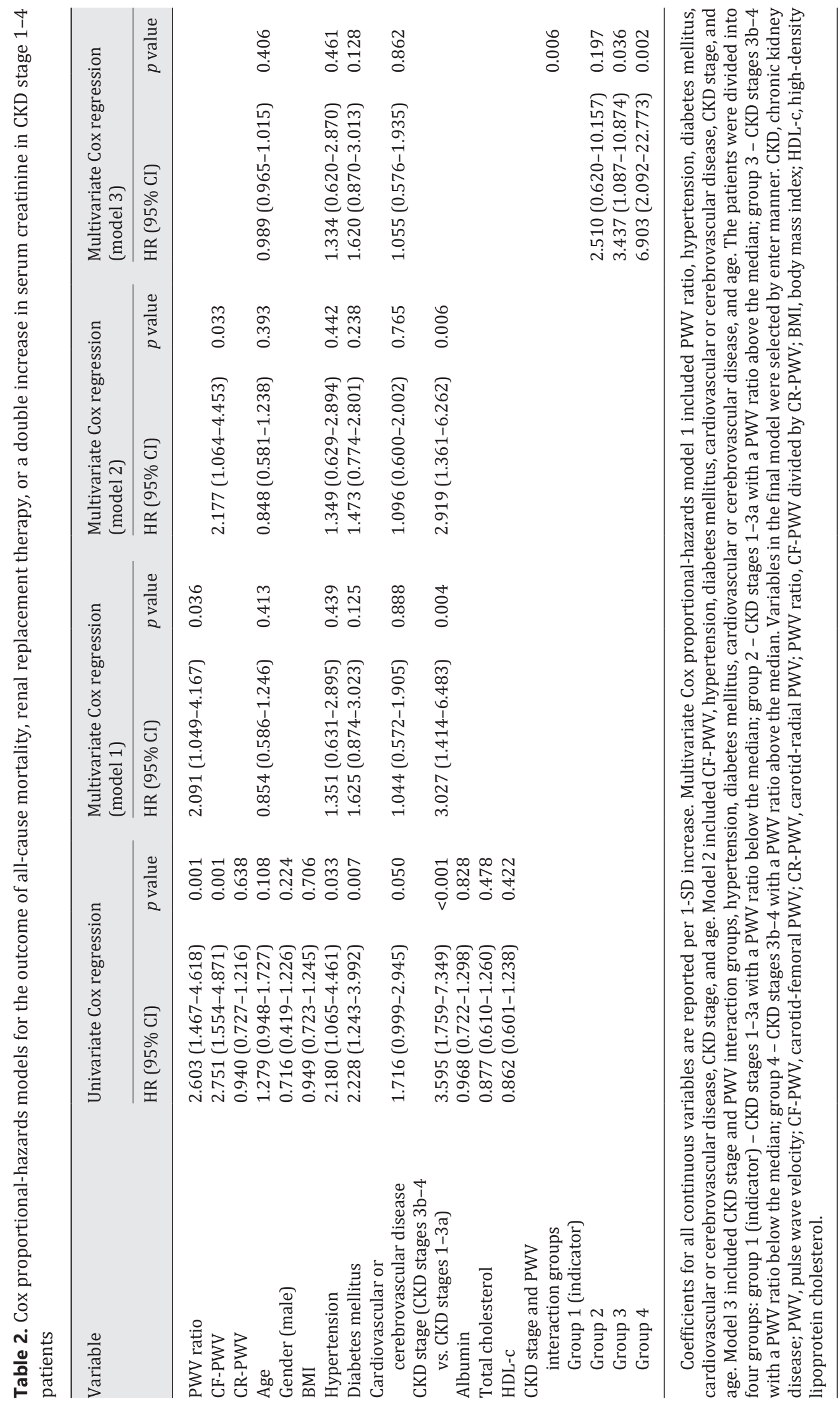




\section{Discussion}

In our investigation, we demonstrated that the aortic-brachial arterial stiffness gradient, defined as the ratio of CF-PWV to CR-PWV, was significantly related to composite outcome of CKD stage 1-4 patients, including all-cause mortality, renal functional deterioration, and RRT. However, the PWV ratio provides no additional predictive value over CF-PWV for CKD stage 1-4 patients. We could not replicate the previous findings that underscored the prognostic importance of the PWV ratio for dialysis patients [21].

Aortic stiffness, which results in increased pulse pressure, cardiac overload, and left ventricular hypertrophy, is an independent predictor of all-cause and cardiovascular death in many populations, as well as of diseases like end-stage renal disease (ESRD), CKD, coronary atherosclerosis, diabetes, and stroke and of aging [30-38]. Moreover, arterial stiffness is linked to a decreased GFR, and is predictive of kidney disease progression [39]. PWV is the most widely used technique. Although PWV can be measured on any artery or between any arterial sites, only CF-PWV has been shown to have predictive value for morbidity and mortality [40]. CF-PWV has been considered as the "gold standard" for measuring aortic stiffness.

Physiologically, the aorta is much more elastic than peripheral muscular arteries, causing an increase in arterial stiffness (stiffness gradient) from the heart to the periphery. This physiological gradient of arterial stiffness results in a gradual attenuation of the forward pressure wave throughout its passage along the arterial tree down to the microcirculation, where pulsatility is minimal. In normal aging, aortic stiffness increases to a greater extent than that of the peripheral muscular arteries, resulting in equalization or even reversal of the stiffness gradient (aortic stiffness > muscular artery stiffness) [41-44], which results in higher pulse pressure transmission into the microcirculation. This leads to a vascular myogenic response, endothelial dysfunction, hypoperfusion, and organ damage. The transmission of excessive forward pressure may be especially detrimental to high-flow organs such as the brain or the kidney. Indeed, at least two studies have shown that increased arterial stiffness by itself is associated with transmission of increased flow pulsatility into the brain and kidneys, leading to structural brain damage, microalbuminuria, and kidney injury $[45,46]$.

The aortic-brachial arterial stiffness gradient is a relatively new concept in arterial research. Fortier et al. [21] reported that in dialysis patients, an increased aortic-brachial arterial stiffness gradient (defined as the ratio of CF-PWV to CR-PWV) was a better predictor of all-cause mortality than CF-PWV. Our recent study also demonstrated that the power of the PWV ratio in predicting both all-cause and CVD mortality was stronger than that of CF-PWV in PD patients [27]. A recent study on CKD stage 3-4 patients demonstrated that reversal of the arterial stiffness gradient is evident starting from stage $3 \mathrm{~b}$ [47]. However, to our knowledge, the predictive value of the PWV ratio for CKD patients' outcome has not yet been evaluated.

In this study, we found that PWV ratio was significantly related to patient outcome in CKD stage 1-4 patients. However, when we compared the predictive value of CF-PWV with that of the PWV ratio for outcome of CKD, the predictive value of CF-PWV was slightly stronger than that of the PWV ratio in these patients. This result is consistent with a recent study that demonstrated that the PWV ratio did not provide any additional prognostic value over conventional CF-PWV in a community cohort [24].

The discrepancy between the predictive value of the PWV ratio for CKD stage 1-4 patients and that for ESRD patients could be explained by several factors. First, the patients included in the present study were CKD stage 1-4 patients, whereas the study by Fortier et al. [21] and our previous study [27] included only dialysis patients - a highly selected group of patients obviously comprising more advanced renal disease patients. Second, this discrepancy could be generated by differences in the definition of clinical outcome, which was all-cause mortality 
in the study by Fortier et al. [21] and CVD mortality in the Framingham Heart Study [24], but a composite outcome of a double increase in serum creatinine, RRT, and death in the present study. Besides, given that reversal of the arterial stiffness gradient becomes evident in CKD patients starting from stage $3 \mathrm{~b}$ [47], the CKD stage 1-3a patients in the present study could have diluted the predictive value of the PWV ratio. We speculate that the differing predictive value of the PWV ratio for CKD and ESRD patients might be explained by the fact that ESRD patients on dialysis have many nontraditional CVD risk factors such as fluid overload, oxidative stress, inflammation, etc., which may exacerbate the loss of the normal gradient in arterial compliance.

Previous studies have shown that hemodialysis itself can affect arterial stiffness levels and thus, consequently, the value of CF-PWV, CR-PWV, and/or CF-PWV/CR-PWV [48, 49]. In the present study, we only included CKD stage 1-4 patients, and all the PWV measurements were taken at baseline. We believe that there is no effect stemming from dialysis per se. In addition, previous works have shown that PWV ratios measured on the side where a vascular access is placed are significantly higher than those obtained on the contralateral side [26]. However, in the present study, none of the patients did undergo any vascular access surgery when measuring PWV. Thus, it was assumed that arterial stiffness should not be different between the left and the right side of the body. Similar to a previous study on hemodialysis patients [50], our study confirmed for the present CKD cohort that PWV ratio was associated with age and diabetes mellitus. However, limited by the data resources, we were unable to investigate the hydration status of the patients in the present cohort. Further study investigating the association of volume status with PWV ratio is needed.

Our study has several strengths that merit comment. For example, it included a relatively large number of CKD patients with sufficient follow-up to assess clinically relevant outcomes, and we were the first to have defined the relationship between PWV ratio and CKD patients' clinical outcome. However, the study also has certain limitations that need to be mentioned. First, the patients included in this study were recruited from a single tertiary academic hospital in China, thereby raising the possibility of ascertainment bias. Second, some detailed management information could not be obtained. Third, the number of CVD deaths was relatively small, which limited our ability to assess the predictive value of the PWV ratio for cardiovascular events and cardiovascular mortality. Fourth, the present study could not quantify beta-PWV, which was reported in recent studies as a way to adjust, in a "subjectspecific" way, the value of PWV considering the subject's blood pressure level [51]. Thus, the intrinsic dependence of PWV on blood pressure could not be ruled out. Finally, although we attempted to adjust for a range of demographic, clinical, and laboratory characteristics, residual confounding remains possible.

In conclusion, our study proved that PWV ratio, as a measure of the aortic-brachial arterial stiffness gradient, was associated with CKD patients' outcome. Patients with advanced CKD and higher PWV ratios had a significantly higher risk of adverse CKD outcome.

\section{Statement of Ethics}

Our research was conducted ethically in accordance with the World Medical Association Declaration of Helsinki. The study protocol was approved by the Peking University Third Hospital Medical Science Research Ethics Committee on human research.

\section{Disclosure Statement}

The authors have no conflicts of interest to declare. 
Bai et al.: PWV Ratio and CKD Outcomes

\section{Funding Sources}

This work was supported by grants from the National Natural Science Foundation of China (project No. 30900681), the Chinese Society of Nephrology (No. 1405046058), and the Fund of the Peking University Health Science Center (BMU20160584). The funders had no role in the study design, data collection, and analysis; decision to publish; or preparation of the manuscript.

\section{Author Contributions}

Qiong Bai was involved in data collection, data analysis, and drafting of the manuscript. Chun-Yan Su was involved in data collection and analysis. Ai-Hua Zhang and Tao Wang were involved in data collection and interpretation of the results. Wen Tang conceptualized the idea, interpreted the results, and wrote part of the draft.

\section{References}

1 Zhang L, Long J, Jiang W, Shi Y, He X, Zhou Z, et al. Trends in Chronic Kidney Disease in China. N Engl J Med. 2016 Sep;375(9):905-6.

2 Bello AK, Levin A, Manns BJ, Feehally J, Drueke T, Faruque L, et al.; Kidney Health for Life Initiative. Effective CKD care in European countries: challenges and opportunities for health policy. Am J Kidney Dis. 2015 Jan; 65(1):15-25.

3 Sarnak MJ, Levey AS, Schoolwerth AC, Coresh J, Culleton B, Hamm LL, et al.; American Heart Association Councils on Kidney in Cardiovascular Disease, High Blood Pressure Research, Clinical Cardiology, and Epidemiology and Prevention. Kidney disease as a risk factor for development of cardiovascular disease: a statement from the American Heart Association Councils on Kidney in Cardiovascular Disease, High Blood Pressure Research, Clinical Cardiology, and Epidemiology and Prevention. Circulation. 2003 Oct;108(17):2154-69.

4 Go AS, Chertow GM, Fan D, McCulloch CE, Hsu CY. Chronic kidney disease and the risks of death, cardiovascular events, and hospitalization. N Engl J Med. 2004 Sep;351(13):1296-305.

5 Muntner P, He J, Hamm L, Loria C, Whelton PK. Renal insufficiency and subsequent death resulting from cardiovascular disease in the United States. J Am Soc Nephrol. 2002 Mar;13(3):745-53.

6 Weiner DE, Tighiouart H, Amin MG, Stark PC, MacLeod B, Griffith JL, et al. Chronic kidney disease as a risk factor for cardiovascular disease and all-cause mortality: a pooled analysis of community-based studies. J Am Soc Nephrol. 2004 May;15(5):1307-15.

7 Adenwalla SF, Graham-Brown MP, Leone FM, Burton JO, McCann GP. The importance of accurate measurement of aortic stiffness in patients with chronic kidney disease and end-stage renal disease. Clin Kidney J. 2017 Aug; 10(4):503-15.

8 Vlachopoulos C, Aznaouridis K, Stefanadis C. Prediction of cardiovascular events and all-cause mortality with arterial stiffness: a systematic review and meta-analysis. J Am Coll Cardiol. 2010 Mar;55(13):1318-27.

9 Ben-Shlomo Y, Spears M, Boustred C, May M, Anderson SG, Benjamin EJ, et al. Aortic pulse wave velocity improves cardiovascular event prediction: an individual participant meta-analysis of prospective observational data from 17,635 subjects. J Am Coll Cardiol. 2014 Feb;63(7):636-46.

10 Rodriguez RA, Shea B, Hae R, Burns KD. The impact of intervention strategies that target arterial stiffness in end-stage renal disease: a systematic review protocol. Syst Rev. 2016 Jul;5(1):118.

11 Zhe XW, Tian XK, Chen W, Guo LJ, Gu Y, Chen HM, et al. Association between arterial stiffness and peritoneal fluid kinetics. Am J Nephrol. 2008;28(1):128-32.

12 Zhe XW, Tian XK, Chen W, Guo LJ, Gu Y, Chen HM, et al. Association between arterial stiffness and peritoneal small solute transport rate. Artif Organs. 2008 May;32(5):416-9.

13 Zhe XW, Zeng J, Tian XK, Chen W, Gu Y, Cheng LT, et al. Pulse wave velocity is associated with metabolic syndrome components in CAPD patients. Am J Nephrol. 2008;28(4):641-6.

14 Lu Q, Cheng LT, Wang T, Wan J, Liao LL, Zeng J, et al. Visceral fat, arterial stiffness, and endothelial function in peritoneal dialysis patients. J Ren Nutr. 2008 Nov;18(6):495-502.

15 Gu Y, Cheng LT, Chen HM, Sun XY, Tang LJ, Guo LJ, et al. Strong association between nutritional markers and arterial stiffness in continuous ambulatory peritoneal dialysis patients. Blood Purif. 2008;26(4):340-6.

$16 \mathrm{Gu} \mathrm{Y,} \mathrm{Cheng} \mathrm{LT,} \mathrm{Zeng} \mathrm{J,} \mathrm{Wang} \mathrm{T.} \mathrm{Increased} \mathrm{arterial} \mathrm{stiffness} \mathrm{in} \mathrm{elderly} \mathrm{female} \mathrm{diabetic} \mathrm{peritoneal} \mathrm{dialysis}$ patients. Am J Nephrol. 2009;29(5):414-9.

17 Tang W, Cheng LT, Lu XH, Wang T. Effect of nutrition on arterial stiffness in peritoneal dialysis patients. Am J Nephrol. 2009;30(2):120-5.

18 Cheng LT, Tang LJ, Chen HM, Tang W, Wang T. Relationship between serum albumin and pulse wave velocity in patients on continuous ambulatory peritoneal dialysis. Vasc Health Risk Manag. 2008;4(4):871-6. 
Briet M, Collin C, Karras A, Laurent S, Bozec E, Jacquot C, et al.; Nephrotest Study Group. Arterial remodeling associates with CKD progression. J Am Soc Nephrol. 2011 May;22(5):967-74.

20 Covic A, Siriopol D. Pulse wave velocity ratio: the new "gold standard" for measuring arterial stiffness. Hypertension. 2015 Feb;65(2):289-90.

21 Fortier C, Mac-Way F, Desmeules S, Marquis K, De Serres SA, Lebel M, et al. Aortic-brachial stiffness mismatch and mortality in dialysis population. Hypertension. 2015 Feb;65(2):378-84.

22 Fortier C, Agharazii M. Arterial Stiffness Gradient. Pulse (Basel). 2016 Apr;3(3-4):159-66.

23 Fortier C, Sidibé A, Desjardins MP, Marquis K, De Serres SA, Mac-Way F, et al. Aortic-Brachial Pulse Wave Velocity Ratio: A Blood Pressure-Independent Index of Vascular Aging. Hypertension. 2017 Jan;69(1):96-101.

24 Niiranen TJ, Kalesan B, Larson MG, Hamburg NM, Benjamin EJ, Mitchell GF, et al. Aortic-Brachial Arterial Stiffness Gradient and Cardiovascular Risk in the Community: The Framingham Heart Study. Hypertension. 2017 Jun;69(6):1022-8.

25 Fortier C, Desjardins MP, Agharazii M. Aortic-Brachial Pulse Wave Velocity Ratio: A Measure of Arterial Stiffness Gradient Not Affected by Mean Arterial Pressure. Pulse (Basel). 2018 Mar;5(1-4):117-24.

26 Bia D, Galli C, Zocalo Y, Valtuille R, Wray S, Pessana F, et al. Central-To-Peripheral Arterial Stiffness Gradient in Hemodialyzed Patients Depends on the Location of the Upper-limb Vascular Access. Curr Hypertens Rev. 2018;14(2):128-36.

27 Bao W, Wang F, Tang W. Aortic-Brachial Stiffness Mismatch and Mortality in Peritoneal Dialysis Patients. Kidney Blood Press Res. 2019;44(1):123-32.

28 Asmar R, Benetos A, Topouchian J, Laurent P, Pannier B, Brisac AM, et al. Assessment of arterial distensibility by automatic pulse wave velocity measurement. Validation and clinical application studies. Hypertension. 1995 Sep;26(3):485-90.

29 Levey AS, Stevens LA, Schmid CH, Zhang YL, Castro AF 3rd, Feldman HI, et al.; CKD-EPI (Chronic Kidney Disease Epidemiology Collaboration). A new equation to estimate glomerular filtration rate. Ann Intern Med. 2009 May;150(9):604-12.

30 Shoji T, Emoto M, Shinohara K, Kakiya R, Tsujimoto Y, Kishimoto H, et al. Diabetes mellitus, aortic stiffness, and cardiovascular mortality in end-stage renal disease. J Am Soc Nephrol. 2001 Oct;12(10):2117-24.

31 Laurent S, Boutouyrie P, Asmar R, Gautier I, Laloux B, Guize L, et al. Aortic stiffness is an independent predictor of all-cause and cardiovascular mortality in hypertensive patients. Hypertension. 2001 May;37(5):1236-41.

32 Guerin AP, Blacher J, Pannier B, Marchais SJ, Safar ME, London GM. Impact of aortic stiffness attenuation on survival of patients in end-stage renal failure. Circulation. 2001 Feb;103(7):987-92.

33 Meaume S, Benetos A, Henry OF, Rudnichi A, Safar ME. Aortic pulse wave velocity predicts cardiovascular mortality in subjects [\{GT\}]70 years of age. Arterioscler Thromb Vasc Biol. 2001 Dec;21(12):2046-50.

34 Cruickshank K, Riste L, Anderson SG, Wright JS, Dunn G, Gosling RG. Aortic pulse-wave velocity and its relationship to mortality in diabetes and glucose intolerance: an integrated index of vascular function? Circulation. 2002 Oct;106(16):2085-90.

35 Boutouyrie P, Tropeano AI, Asmar R, Gautier I, Benetos A, Lacolley P, et al. Aortic stiffness is an independent predictor of primary coronary events in hypertensive patients: a longitudinal study. Hypertension. 2002 Jan; 39(1):10-5.

36 Laurent S, Katsahian S, Fassot C, Tropeano AI, Gautier I, Laloux B, et al. Aortic stiffness is an independent predictor of fatal stroke in essential hypertension. Stroke. 2003 May;34(5):1203-6.

37 Najjar SS, Scuteri A, Lakatta EG. Arterial aging: is it an immutable cardiovascular risk factor? Hypertension. 2005 Sep;46(3):454-62.

38 Karras A, Haymann JP, Bozec E, Metzger M, Jacquot C, Maruani G, et al.; Nephro Test Study Group. Large artery stiffening and remodeling are independently associated with all-cause mortality and cardiovascular events in chronic kidney disease. Hypertension. 2012 Dec;60(6):1451-7.

39 Briet M, Boutouyrie P, Laurent S, London GM. Arterial stiffness and pulse pressure in CKD and ESRD. Kidney Int. 2012 Aug;82(4):388-400.

40 Reference Values for Arterial Stiffness' Collaboration. Determinants of pulse wave velocity in healthy people and in the presence of cardiovascular risk factors: 'establishing normal and reference values'. Eur Heart J. 2010 Oct;31(19):2338-50.

41 Avolio AP, Chen SG, Wang RP, Zhang CL, Li MF, O'Rourke MF. Effects of aging on changing arterial compliance and left ventricular load in a northern Chinese urban community. Circulation. 1983 Jul;68(1):50-8.

42 McEniery CM, Yasmin, Hall IR, Qasem A, Wilkinson IB, Cockcroft JR; ACCT Investigators. Normal vascular aging: differential effects on wave reflection and aortic pulse wave velocity: the Anglo-Cardiff Collaborative Trial (ACCT). J Am Coll Cardiol. 2005 Nov;46(9):1753-60.

43 Mitchell GF, Parise H, Benjamin EJ, Larson MG, Keyes MJ, Vita JA, et al. Changes in arterial stiffness and wave reflection with advancing age in healthy men and women: the Framingham Heart Study. Hypertension. 2004 Jun;43(6):1239-45.

44 Mitchell GF, Wang N, Palmisano JN, Larson MG, Hamburg NM, Vita JA, et al. Hemodynamic correlates of blood pressure across the adult age spectrum: noninvasive evaluation in the Framingham Heart Study. Circulation. 2010 Oct;122(14):1379-86.

45 Mitchell GF, van Buchem MA, Sigurdsson S, Gotal JD, Jonsdottir MK, Kjartansson Ó, et al. Arterial stiffness, pressure and flow pulsatility and brain structure and function: the Age, Gene/Environment SusceptibilityReykjavik study. Brain. 2011 Nov;134(Pt 11):3398-407. 
46 Hashimoto J, Ito S. Central pulse pressure and aortic stiffness determine renal hemodynamics: pathophysiological implication for microalbuminuria in hypertension. Hypertension. 2011 Nov;58(5):839-46.

47 Zanoli L, Lentini P, Boutouyrie P, Fatuzzo P, Granata A, Corrao S, et al. Pulse wave velocity differs between ulcerative colitis and chronic kidney disease. Eur J Intern Med. 2018 Jan;47:36-42.

48 Bia D, Galli C, Zócalo Y, Valtuille R, Wray S, Armentano R, et al. Hemodialysis Decreases the EtiologicallyRelated Early Vascular Aging Observed in End-Stage Renal Disease: A 5-Year Follow-Up Study. Blood Purif. 2017;43(1-3):18-30.

49 Cabrera Fischer EI, Bia D, Galli C, Valtuille R, Zócalo Y, Wray S, et al. Hemodialysis decreases carotid-brachial and carotid-femoral pulse wave velocities: a 5-year follow-up study. Hemodial Int. 2015 Jul;19(3):419-28.

50 Bia D, Valtuille R, Galli C, Wray S, Armentano R, Zócalo Y, et al. Aortic-Radial Pulse Wave Velocity Ratio in Endstage Renal Disease Patients: Association with Age, Body Tissue Hydration Status, Renal Failure Etiology and Five Years of Hemodialysis. High Blood Press Cardiovasc Prev. 2017 Mar;24(1):37-48.

51 Spronck B, Delhaas T, Butlin M, Reesink KD, Avolio AP. Options for Dealing with Pressure Dependence of Pulse Wave Velocity as a Measure of Arterial Stiffness: An Update of Cardio-Ankle Vascular Index (CAVI) and CAVI0. Pulse (Basel). 2018 Mar;5(1-4):106-14. 